\title{
137. Supratentorial Tumors in Infancy and Childhood
}

- Clinical Survey on 51 Cases -

\author{
Katsutoshi Kitamura, Yasuhiko Matsukado, Masamichi Tomonaga, \\ Kazuo Kinoshita, Yukichi IKawa, Kenichi Nishimura \\ and Toshio SAWADA \\ First Department of Surgery, Faculty of Medicine, Kyushu \\ University, Fukuoka, Japan \\ (Director: Prof. H. Miyake)
}

\begin{abstract}
Supratentorial tumors in childhood have been much more frequently encountered in our Department than shown in many other statistic surveys. Out of about 100 intracranial space taking lesions in patients below the age of 16 years treated in our Department, 51 were supratentorial lesions. They were neoplastic in 36 cases ( 11 gliomas, 10 pineal tumors, 8 craniopharyngiomas, 3 meningiomas, 2 pituitary tumors and 2 metastatic tumors) and non-neoplastic tumors in 15 cases ( 1 cyst of cerebral hemisphere, 3 brain abscesses, 4 cerebral paragonimiases, 4 chronic subdural hematomas, and 3 chronic subdural hygromas).

In patients below the age of 8 years gliomas of cerebral hemisphere were common, while above 8 years, pineal body tumors and craniopharyngiomas predominated. Meningiomas and pituitary tumors were seen only in a small incidence. Signs and symptoms such as headache or papilledema were usually less significant than in adult, and focal signs also appeared only in late stage in many cases. Thus the tumors exposed were often far more extensive than expected before the operation. This seem mainly due to the skull in infancy yielding to the increased intracranial pressure. Abnormal enlargement of the head in infancy should be emphasized to be one of the most important signs in the disorders above mentioned.
\end{abstract}

\section{Cytological Studies on the Atypical Cell in the Circulating Blood and the Cerebrospinal Fluid of the Patients with Malignant Tumor}

\author{
Yozo Aoyagi, M.D., Mamoru Takagaki, M.D., Kimiharu Furusawa, \\ M.D. and Seiji Hosoda, M.D. \\ Dept. of 2nd Surgery, Juntendo Univ. School of Medicine
}

To find the tumor cells in the circulating blood and the spinal fluid, authors have studied cytologically 22 specimens of the patients with brain tumor. 